06

\title{
Модифицированный метод $z$-сканирования для определения нелинейности третьего порядка для квантовых точек в режиме резонансного возбуждения
}

\author{
(С) Е.А. Пономарева, И.Д. Скурлов, С.Э. Путилин, А.Н. Цыпкин, А.П. Литвин
}

Университет ИТМО,

197101 Санкт-Петербург, Россия

"e-mail: litvin88@gmail.com

Поступила в редакцию 10.10.2018 г.

\begin{abstract}
Предложен модифицированный метод $z$-сканирования для определения нелинейного показателя преломления в ближнем инфракрасном спектральном диапазоне для коллоидных квантовых точек в режиме резонансного возбуждения. Показано, что за счет уменьшения частоты повторения импульсов можно избежать термических эффектов, связанных с нагревом раствора из-за сильного поглощения света. Продемонстрировано, что нелинейный показатель преломления растворов с квантовыми точками сульфида свинца имеет порядок $10^{-16} \mathrm{~cm}^{2} / \mathrm{W}$.
\end{abstract}

DOI: $10.21883 /$ OS.2019.02.47205.297-18

\section{Введение}

Исследованием нелинейных свойств материалов занимаются с 1960-х годов. Знания о таких свойствах материала позволяют использовать их в различных применениях науки и техники: в наноэлектронике, в быстрых оптических фотоприемниках, модуляторах и фильтрах. Многообещающими для использования в нелинейнооптических приложениях являются полупроводниковые квантовые точки (КТ) [1]. Ввиду эффекта квантового конфайнмента оптические переходы в КТ сосредоточены в узком интервале энергий, тем самым усиливая резонансный нелинейный отклик таких материалов [2]. КТ сульфида свинца обладают широкой полосой поглощения вплоть до ближнего инфракрасного диапазона, что открывает возможность получения больших нелинейностей в резонансном режиме возбуждения [3]. Большое значение боровского радиуса $(18 \mathrm{~nm})$ для $\mathrm{PbS}$ позволяет исследовать нелинейные свойства КТ различного размера в режиме строгого конфайнмента.

Одной из техник для исследования нелинейно-оптических параметров (в том числе нелинейного показателя преломления, НПП) является метод $z$-сканирования [4,5]. Различают два режима z-сканирования: нерезонансный, при котором возбуждающее излучение не попадает в полосу поглощения, и резонансный, при котором возбуждающее излучение поглощается. При резонансном $z$-сканировании для коллоидных КT PbS были получены следующие значения НПП: $-(10-60) \cdot 10^{-12} \mathrm{~cm}^{2} / \mathrm{W}(d=5.9 \mathrm{~nm})$ в тетрахлорэтилене [6], $-(0.5-4.2) \cdot 10^{-15} \mathrm{~cm}^{2} / \mathrm{GW}$ (в диапазоне размеров от 11 до $4.5 \mathrm{~nm})$ в гексане [7] и $-(3.0 \pm 0.9) \cdot 10^{-14} \mathrm{~cm}^{2} / \mathrm{W} \quad$ в $\mathrm{CS}_{2} \quad[8]$. Сравнение НПП КТ $\mathrm{PbS}(d=9.2 \mathrm{~nm})$ в различных растворителях проведено в [9]; полученные значения составили $-8.2 \cdot 10^{-16} \mathrm{~cm}^{2} / \mathrm{W}$ в толуоле, $-2.3 \cdot 10^{-15} \mathrm{~cm}^{2} / \mathrm{W}$ в гексане, $-1.2 \cdot 10^{-15} \mathrm{~cm}^{2} / \mathrm{W}$ в тетрахлорметане. Для КТ, помещенных в цеолитные матрицы, значение НПП составило $-(11-294) \cdot 10^{-12} \mathrm{~cm}^{2} / \mathrm{W}(d=3-8 \mathrm{~nm})$ в зависимости от концентрации КТ в образце [10].

При исследовании нелинейных свойств КТ в резонансном режиме необходимо учитывать имеющийся температурный вклад в изменение НПП, возникающий вследствие нагрева образца при поглощении высокоинтенсивного лазерного излучения. До сих пор не разработано универсальной методики учета термического вклада в НПП, тем не менее существует один общий момент - использование сверхкоротких лазерных импульсов в качестве зондирующего излучения. Конечные варианты решения проблемы варьируются от работы к работе. В частности, в [6] уход от термического изменения НПП осуществлялся с помощью модуляции зондирующего лазерного излучения (частота следования импульсов $f=10 \mathrm{MHz}$, длительность импульса $\tau=2.5 \mathrm{ps}$ ) затвором шириной $2 \mathrm{~ms}$ и скважностью $7.3 \%$. В работе [7] для учета термальных эффектов снижали мощность лазерного излучения, не меняя другие параметры $(f=1 \mathrm{kHz}$, длительность импульса $\tau=300 \mathrm{fs})$. В работах $[9,10]$ термический вклад в НПП не учитывался. В большинстве упомянутых выше работ использовались лазеры с высоким значением частоты повторения импульсов. Однако при высокой частоте следования импульсов (более $1 / t_{c}=4 D / w^{2}$, где $D-$ коэффициент температуропроводности, $w$ - диаметр лазерного пучка) исследуемый образец не успевает вернуться в состояние температурного равновесия до прихода следующего импульса возбуждения, что способствует накоплению эффекта термической линзы $[11,12]$; для типичной схемы $z$-сканирования данный эффект проявляется при частотах следования, превышающих несколько десятков $\mathrm{kHz}$. 
В приведенных выше работах наблюдалась сильная зависимость НПП от концентрации КТ, что может быть проявлением термической нелинейности. В нашей работе предложен метод, позволяющий избегать влияния температурных эффектов.

\section{Методы исследования}

В настоящей работе предлагается модификация стандартного метода $z$-сканирования, заключающаяся в использовании источника излучения с фемтосекундной длительностью импульса и частотой повторения импульсов до $1 \mathrm{~Hz}$. Снижение до такой частоты гарантированно позволяет избежать накопления термических эффектов, время релаксации которых становится значительно меньше времени между импульсами накачки. Используя такую методику, можно исследовать нелинейные эффекты, имеющие чисто электронную природу.

Экспериментальная установка представляет из себя типичную схему z-сканирования, принципиальная схема на рис. 1. В качестве излучения накачки используется фемтосекундный лазер Ti:Sapphire (Avesta, Россия) со следующими параметрами: центральная длина волны $800 \mathrm{~nm}$, частота повторения импульсов $1 \mathrm{~Hz}$, длительность импульсов от $35 \mathrm{fs}$, энергия в импульсе от 0.1 до $10 \mu \mathrm{J}$. Излучение фокусируется линзой с фокусным расстоянием $20 \mathrm{~cm}$, диаметр перетяжки пучка составил $230 \mu \mathrm{m}$. Регистрация прошедшего излучения происходит с помощью фотоприемников Ophir (Израиль), синхронизированных с лазером и ПК.

Исследуемый образец представляет собой коллоидный раствор КТ $\mathrm{PbS}(d=4.5 \mathrm{~nm})$ в гексане, находящийся в кювете из оптического стекла, прозрачной на длине волны используемого лазерного излучения $(800 \mathrm{~nm})$. Спектр поглощения исследуемых КТ приведен на рис. 2, $a$.

Учет нелинейно-оптических эффектов растворителя и кюветы проводился согласно методу, предложенному в работе [13]. Для каждого образца проводилось предварительное измерение чистого гексана в кювете, в которой проводилось измерение исследуемого раствора. Типичные результаты $z$-сканирования представлены на

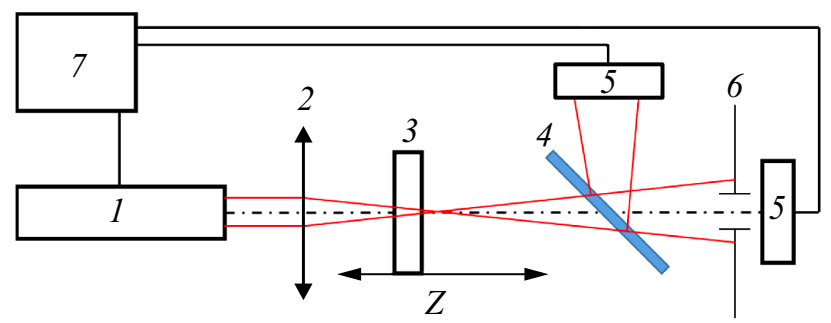

Рис. 1. Экспериментальная установка измерения нелинейного показателя преломления для растворов с КТ. 1 - лазер, $2-$ фокусирующая линза, 3 - подвижный позиционер с кюветой с образцом, 4 - делитель пучка, 5 - фотодетекторы, 6 диафрагма, 7 - ПК.
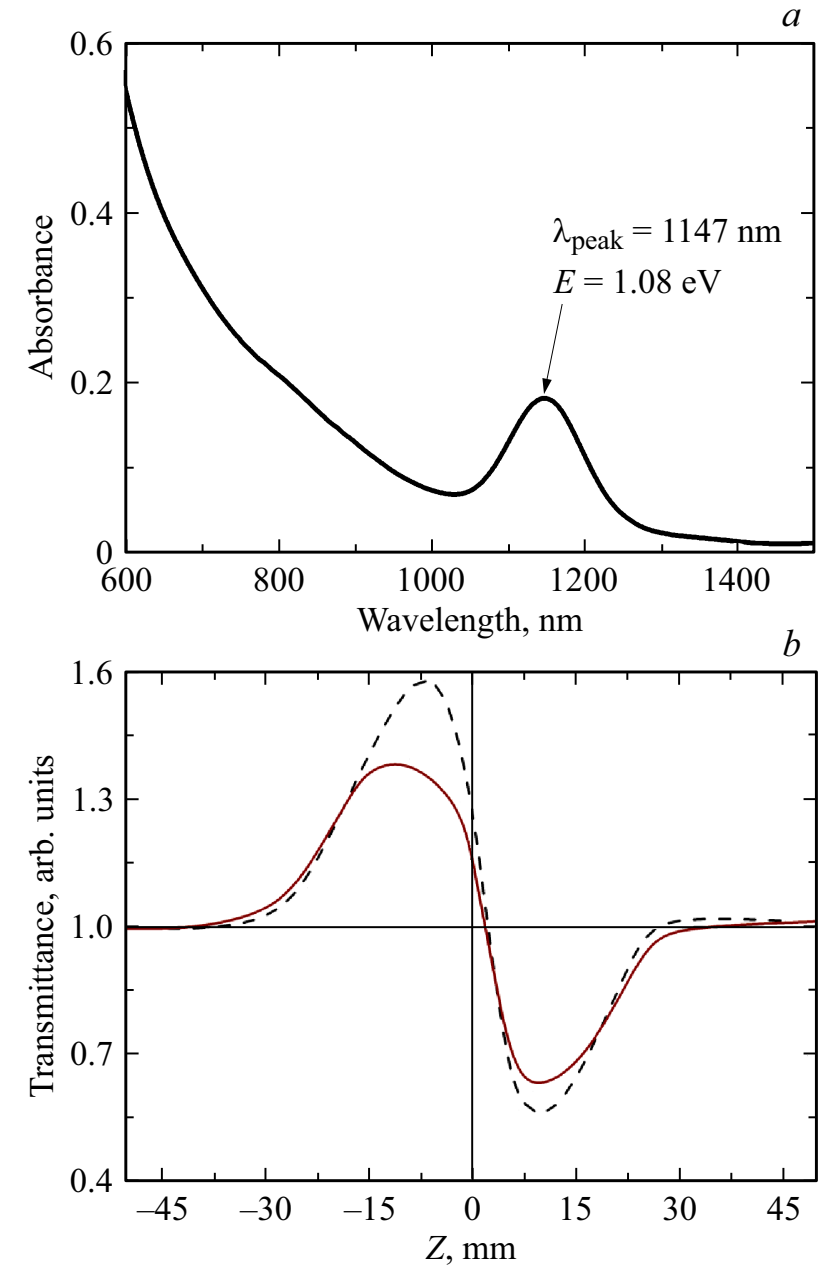

Рис. 2. (a) Спектр поглощения исследуемых КT PbS; (b) Типичные кривые пропускания при $z$-сканировании для чистого растворителя - гексана (штриховая линия) и для раствора КТ $\mathrm{PbS}$ в нем (сплошная линия).

рис. 2, $b$. В предположении об аддитивности НПП растворителя и КТ разность нелинейного пропускания раствора КТ и растворителя позволяет определить вклад, вносимый только кубической нелинейностью КT PbS.

\section{Результаты экспериментов}

Расчет НПП $\left(n_{2}\right)$ проводился по следующей формуле [5]:

$$
n_{2}=\frac{\Delta T}{0.406 I_{\text {in }}} \frac{\sqrt{2} \lambda}{\left(2 \pi L_{\alpha}(1-S)^{0.25}\right)},
$$

где $S$ - апертура диафрагмы, $L-$ толщина образца, $L_{\alpha}-$ эффективная длина взаимодействия $L_{\alpha}=$ $=[1-\exp (-\alpha L)] / \alpha, \lambda-$ длина волны излучения, $\alpha-$ коэффициент поглощения среды, $\Delta T-$ разница между нормированной интенсивностью максимума и минимума на кривой $z$-сканирования.

Во всех случаях КТ дают отрицательный вклад в изменение пропускания, что говорит о наличии процесса 


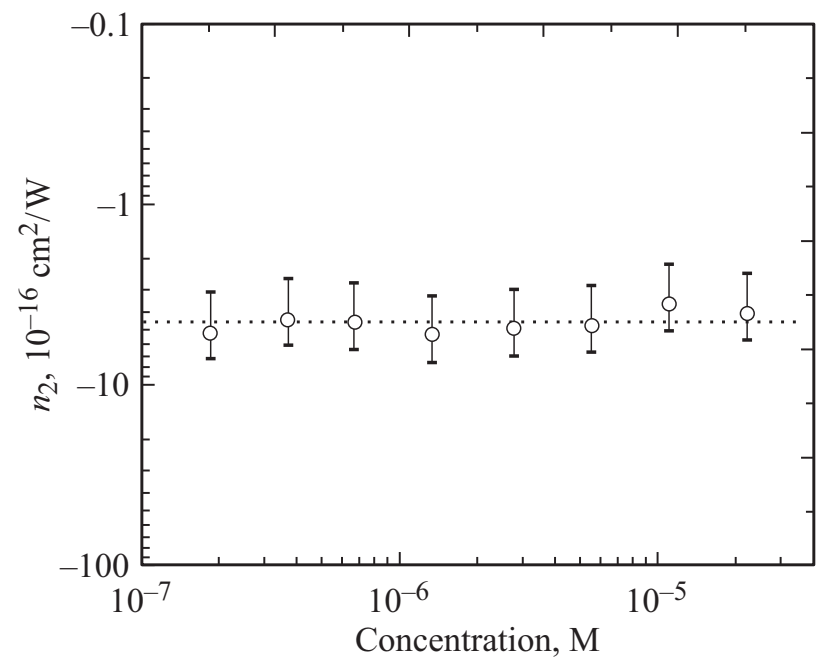

Рис. 3. Зависимость величины НПП от концентрации КТ в гексане. Пунктирная линия отображает среднее значение $-4.55 \cdot 10^{-12} \mathrm{~cm}^{2} / \mathrm{W}$.

самодефокусировки, а следовательно, отрицательного НПП. Зависимость НПП от концентрации КТ в растворе представлена на рис. 3 .

Отметим также, что полученные значения $n_{2}$ порядка $10^{-16} \mathrm{~cm}^{2} / \mathrm{W}$, что на 4 порядка меньше, чем для результатов, представленных в работах [6-10]. С учетом погрешности, эти значения не зависят от концентрации КТ. Оба факта, скорее всего, связаны с отсутствием термического вклада в НПП.

\section{Заключение}

За счет снижения частоты следования зондирующих импульсов пропадает термический вклад в НПП, что позволяет измерять параметры нелинейности третьего порядка для КТ в режиме резонансного возбуждения. Показано, что значение НПП КТ $\mathrm{PbS}$ в отсутствие температурных эффектов на 4 порядка меньше, чем имеющиеся в литературе значения.

Работа выполнена в рамках проекта РФФИ № 18-3200027.

\section{Список литературы}

[1] Banfi G.P., Degiorgio V., Ricard D. // Advances in Physics. 1998. V. 47. N 3. P. 447-510.

[2] Wang Y. // Accounts of Chemical Research. 1991. V. 24. N 5. P. $133-139$.

[3] Yoffe A.D. // Advances in Physics. 1993. V. 42. N 2. P. $173-262$.

[4] Sheik-Bahae M., Said A.A., Wei T., Hagan D.J., van Stryland E.W. // IEEE J.Quantum Electronics. 1990. V. 26. N 4. P. $760-769$.

[5] Cheung Y.M., Gayen S.K. // J. Opt. Soc. Am. B. 1994. V. 11. N 4. P. 636-643.
[6] Omari A., Moreels I., Masia F., Langbein W., Borri P., Thourhout D., Kockaert P., Hens Z. // Phys. Rev. B. 2012. V. 85. N 11. P. 115318.

[7] Neo M.S., Venkatram N., Li G.S., Chin W.S., Ji Wei // The J. Phys. Chem. C. 2009. V. 114. N 42. P. 18037-18044.

[8] Bolotin I.L., Asunskis D.J., Jawaid A.M., Liu Y., Snee P.T., Hanley L. // The J. Phys. Chem. 2010. V. 114. N 39. P. 16257-16262.

[9] Hui Cheng, Yuhua Wang, Hongwei Dai, Jun-Bo Han, Xianchang Li // The J. Phys. Chem.. 2015. V. 119. N 6. P. 3288-3292.

[10] Kim H.S., Yoon K.B. // Coordination Chem. Rev. 2013. V. 263.

[11] Hayes J.N. // Appl. Opt. 1972. V. 11. N 2. P. 455-461.

[12] Falconieri M. // J. Opt. A: Pure Appl. Opt. 1999. V. 1. N 6. P. 662.

[13] Khoroshikh D.A., Tsypkin A.N., Mel'nik M.V., Czingel K., Kokenyesi S. // Opt. Spectrosc. 2016. V. 121. N 5. P. 786-788. 